\title{
Exploratory Factor Analysis of Gait Recognition
}

\author{
Imed Bouchrika \& Mark S. Nixon \\ Department of Electronics and Computer Science \\ University of Southampton, SO17 1BJ, UK \\ \{ib04r, msn\}eecs.soton.ac.uk
}

\begin{abstract}
Many studies have now shown that it is possible to recognize people by the way they walk. As yet there has been little formal study of the effects of covariates on the recognition process. We show how these factors can separately affect the walking pattern. Further we assess the contribution and discriminatory significance of the gait dynamics used for recognition. Based on a covariate-based probe dataset of 440 samples, a high recognition rate of $73.4 \%$ is achieved using the KNN classifier. This is to confirm that people identification using dynamic gait features is still perceivable with better recognition rate even under the different covariate factors.
\end{abstract}

\section{Introduction}

Surveillance technology is of increasing us in modern society. This is largely due to the vital need to provide a safer environment. Because of the rapid growth of security cameras and need for automated analysis, the deployment of biometric technologies becomes important for the development of automated visual surveillance systems. The suitability of gait recognition for surveillance systems emerges from the fact that gait can be perceived from a distance as well as its non-invasive nature. Although gait recognition is still a new biometric and is not sufficiently mature to be deployed in real world applications such as visual surveillance, it overcomes most of the limitations that other biometrics suffer from such as face, fingerprints and iris recognition which can be obscured in most situations where serious crimes are involved.

Gait is a new biometric with a number of benefits arising from its non-intrusive nature and the possibility of use at a distance $[21,3,7,9,10]$. Hence, the analysis of the different covariate factors becomes essential to quantify the intrusiveness of gait recognition which will be the focus of this paper. The covariate factors can be related either to the subject as for the case when a subject smiles for face recognition, or related to the environmental conditions such as lighting, nature of the ground or camera setup. Gait is also affected by different covariate factors including footwear, clothing, injuries, age, walking speed, and much more akin with other biometrics. In fact, the effects of the different covariates for gait analysis and recognition have not been investigated much by medical and other researchers [10], This is mainly due to the lack of availability for databases, as well as the availability of automated systems which would help for the extraction of gait features. Moreover, the complexity of earlier model-based approaches has precluded their deployment for this analysis.

The effects of covariate factors on the performance of gait recognition using computer vision methods have been investigated by only one recent major research study by Sarkar et al. [15]. Sarker described a baseline algorithm for gait recognition based on the temporal correlation of silhouette data. The algorithm is evaluated on a set of twelve experiments in order to examine the effects of the different covariates including viewpoint, footwear, walking surface, time and carrying conditions. However, their work lacks exploratory analysis of the different gait features under covariate data due to the use of the silhouette approach. In this research, a full investigation is carried out to explore the covariate effects on gait recognition using dynamic-related features derived via model-based method. The covariate factors includes footwear, clothing, carrying conditions and walking speed. Furthermore, we assess the contribution and discriminatory significance of the different dynamic ( gaitrelated ) features used for gait recognition. The previous covariate studies [15] have used the NIST data which combines covariates. We now study the independent effect of covariates using the SOTON covariate database where only one covariate is changed between sequences ( except time which affects all sequences equally).

\section{Data Acquisition for Covariate Analysis}

In order to study the exploratory effects of covariate factors on gait recognition, a gallery dataset of 160 video sequences is taken from the SOTON gait database. The galley consists of 20 different walking subjects with 8 sequences 
for every individual recorded without covariate effects. Further, a probe dataset of 440 video sequences is collected from the Southampton Covariate Database. The dataset consists of ten different walking subjects with eight males and two females. Each subject is recorded from the sagittal view walking at eleven different scenarios, including normal walking. Four video sequences are taken for each situation. The different recorded scenarios are aimed to investigate the following factors:

- Footwear: flip-flop, trainer, bare-feet, boots.

- Clothing: coat, trench coat.

- Carrying Conditions: barrel bag, handbag.

- Walking Speed: normal, quick and slow walking.

To extract the gait features of walking subjects from the covariate dataset, we applied the model-based method described in [4] to automate the extraction process of the joint trajectories. Spatial motion templates describing the motion of the joints are derived by manual gait analysis and used to aid the markerless extraction of the joint positions. A recursive evidence gathering algorithm is employed for the extraction process whereby spatial model templates for the human motion are presented in a parameterized form using the Elliptic Fourier Descriptors described in equation (1):

$\left[\begin{array}{l}x(t) \\ y(t)\end{array}\right]=\left[\begin{array}{l}a_{0} \\ b_{0}\end{array}\right]+\left[\begin{array}{cc}\cos (\alpha) & -\sin (\alpha) \\ \sin (\alpha) & \cos (\alpha)\end{array}\right]\left[\begin{array}{c}F_{x}(t) * s_{x} \\ F_{y}(t) * s_{y}\end{array}\right]$

where $\alpha$ is the rotation angle, $s_{x}$ and $s_{y}$ are the scaling factors across the horizontal and vertical axes respectively. $a_{0}$ and $b_{0}$ define the position of the shape's centre. $F_{x}(t)$ and $F_{y}(t)$ are computed using equation :

$$
\begin{aligned}
& F_{x}(t)=\sum_{k=1}^{n} a_{x_{k}} \cos (k t)+b_{x_{k}} \sin (k t) \\
& F_{y}(t)=\sum_{k=1}^{n} a_{y_{k}} \cos (k t)+b_{y_{k}} \sin (k t)
\end{aligned}
$$

where $a_{x_{k}}, a_{y_{k}}, b_{x_{k}}$ and $b_{y_{k}}$ are the set of the elliptic coffecients which can be computed by a Riemann summation [1]. Gait knowledge is exploited via heel strike extraction to reduce the the parameter space dimensionality and therefore reduce the computational load of the evidence gathering algorithm being used in the extraction process.

In order to identify a subject by their gait, we derive the angular measurements as well as the trunk spatial displacement which best describe the gait kinematics. The use of angular motion is very common in gait analysis and recognition. The angles of the joints including the hip and the knee; are considered the most important kinematics of the lower limbs. Feature selection is employed to derive as many discriminative cues as possible whilst removing the redundant and irrelevant gait features which may degrade the recognition rate. It is practically infeasible to run an exhaustive search for all the possible combinations of features in order to obtain the optimal subset for recognition due to the high dimensionality of the feature space. For this reason, we employed the Adaptive Sequential Forward Floating Selection (ASFFS) search algorithm [16]. The algorithm uses a validation-based evaluation criterion which is proposed to find the subset of features that minimises the classification errors as well as ensure good separability between the different classes. In contrast to the voting scheme used in the $\mathrm{KNN}$, the evaluation function uses different weights $w$ to signify the importance of the most nearest neighbours. The probability score for a sample $s_{c}$ to belong to class $c$ is expressed in the following equation (3):

$$
f\left(s_{c}\right)=\frac{\sum_{i=1}^{N_{c}-1} z_{i} w_{i}}{\sum_{i=1}^{N_{c}-1} w_{i}}
$$

where $N_{c}$ is the number of instances in class $c$, and the weight $w_{i}$ for the $i^{t h}$ nearest instance is inversely related to proximity as:

$$
w_{i}=\left(N_{c}-i\right)^{2}
$$

The value of $z_{i}$ is defined as:

$$
z_{i}=\left\{\begin{array}{cc}
1 & \text { if nearest }\left(s_{c}, i\right) \in c \\
0 & \text { otherwise }
\end{array}\right.
$$

such that the nearest $\left(s_{c}, i\right)$ function returns the $i^{\text {th }}$ nearest instance to the sample $s_{c}$. The Euclidean distance metric is employed to find the nearest neighbours.

\section{Covariate Analysis for Gait Recognition}

In order to quantity the covariate effects on the performance of gait recognition, the Correct Classification Rate (CCR) is computed using the $K$-nearest neighbour (KNN) classifier with the Leave-one-out cross-validation rule. Based on the subset of features derived using the Feature Selection algorithm, we have achieved a high recognition rate of $95.75 \%$ for the value of $k=5$ using the set of 160 video sequences from the covariate-free dataset. This is achieved using solely features describing purely the dynamics of the locomotion process. Furthermore, we have probed 440 samples from the covariate dataset against the gallery database. A recognition rate of $73.4 \%$ is achieved for all the covariate factors including footwear, clothing, load carriage and walking speed which is higher when compared to the low recognition rates reported by Phillips et al. [15] using the silhouette-based method.

The Cumulative Match Score curves showing the comparative results are shown in Figure (3). Phillips reported a CCR of $57 \%$ for Data (I) with load carriage and footwear covariates whilst a CCR of $3 \%$ is achieved for Data (II) 


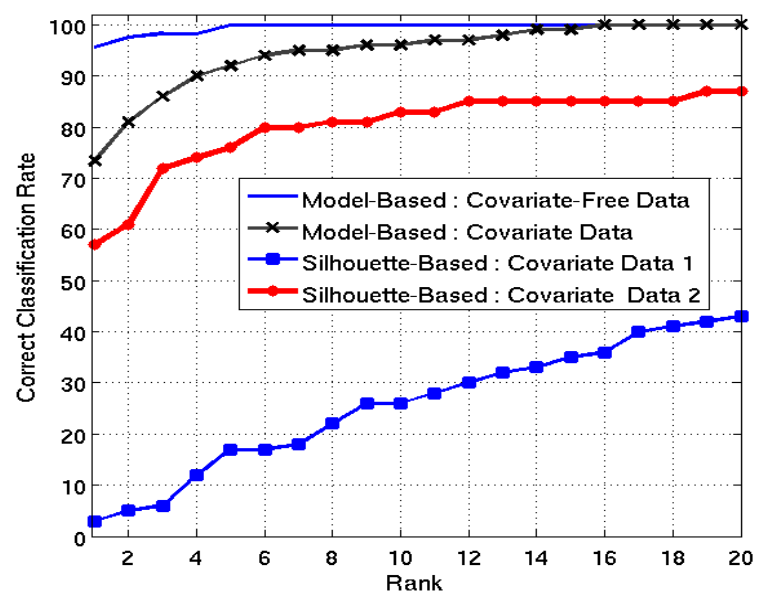

Figure 1. The Cumulative Match Score Curves for the Classification Results.

with the following covariates : time, footwear, and clothing. Time has been shown $[15,19]$ to play a major part in reducing recognition capability by gait. Using a silhouette based approach Veres showed that this could be redressed by fusing those parts of the gait signature which are invariant with time. In this way the overall CCR could be improved from 23 to $27 \%$ [20]. By modelling the change in feature space (by using linear interpolation) the recognition rate with variation in time was improved from $23 \%$ to $65 \%$ [19]. Both of these are considerably improved over the 3\% achieved by Phillips et al [15]. Given the limited data on time, Veres' study and the depth of her results, the time factor is included implicitly and not considered further here.

\subsection{The Footwear Effects}

The gait pattern is affected by the different footwear as people are observed to walk differently when wearing trainers as to when wearing flip flops. This has been confirmed by research carried out by Dobbs et al. [5]. Based on their experimental results, it was reported that the stride and cadence parameters of the walking pattern are affected by footwear as opposed to walking with barefeet. Moreover, recent studies [11] showed that changing the footwear texture causes changes in the gait pattern. In the studies carried out by Phillips et al. [15] to investigate the footwear effects on the performance of gait recognition, a high recognition rate of $78 \%$ is reported using a silhouette-based method. This is because of the fact that body-related or silhouettebased features are almost invariant to the different footwear.

In order to explore the effects of footwear on the performance of people identification using dynamic gait features, a number of experiments are carried out for subjects wearing a variety of different footwear including flip flop, boots and normal shoes. In addition, subjects are also recorded walking with barefeet. For each of the footwear-related factors, 40 video sequences are processed to derive gait signatures based on the dynamic gait features. To assess the classification performance, subjects are validated against the gallery dataset which consists of 160 gait signatures for 20 different subjects recorded with no covariate effects.

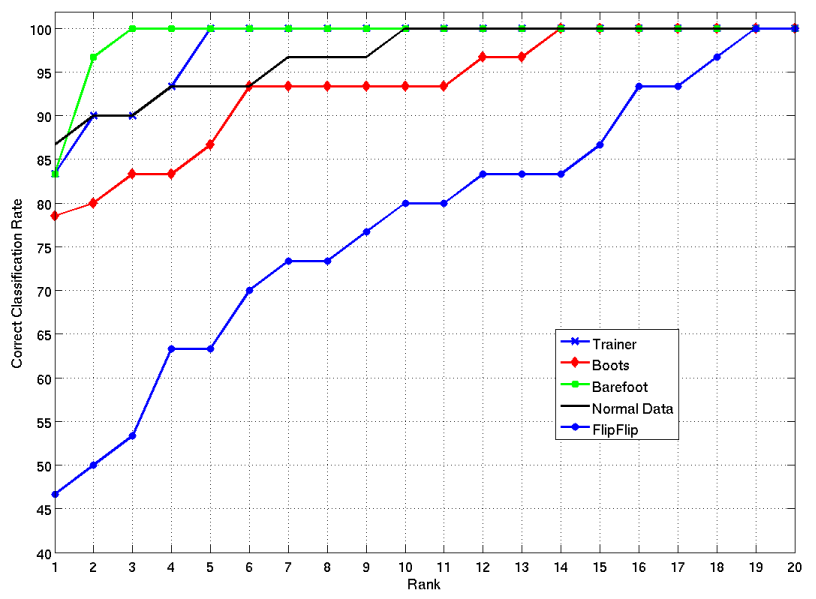

Figure 2. Classification Results for the Footwear Covariates.

The classification results for the footwear covariates are expressed using the cumulative match score as shown in Figure (2). The recognition rates for the trainer and boots cases are observed to be almost the same as the normal case with achieved rates of $78 \%, 83.33 \%$ and $86.67 \%$ for the boots, trainer and normal shoe cases respectively. When subjects are assessed walking with barefeet, the same gait recognition rate is achieved as the other footwear factors including trainers, boots and normal shoes with a reported CCR of $83.33 \%$. This suggests that the dynamic gait features for people identification are not affected largely with the different footwear. However, the human gait is observed to vary much when people walk with flip flops as the recognition rate drops largely to $46 \%$. This is likely due to the comfortability issue with flip flops which are not commonly to worn in the UK, and the mode grip differs in that the pillar in the flip flop is clenched between the big toe and its neighbour. Further, there is no rear part of the shoe so this must be compensated when walking.

\subsection{The Clothing Effects}

The clothing effects on human gait as well as the posture and balance can be considerably important. In [13], Punakallio et al showed that suits wore by fire-fighters have significantly impaired their postural and functional balance. In another study by Egan et al [6], it was revealed that clothing properties such as weight can be another factor which have effects on balance and gait of people. Furthermore, Rahmatalla et al [14] concluded that restrictive clothing can 
impose constraints on the relative joint angle limits of the walking subject and therefore affect their gait pattern. In the study carried out by Phillips et al. [15] for gait recognition using the silhouette-based approach, the recognition rate dropped sharply to $3 \%$ for the following combined covariate factors: time, footwear and clothing.

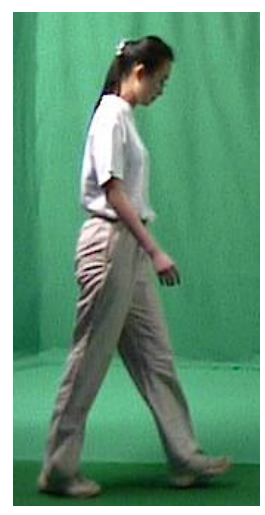

(a)

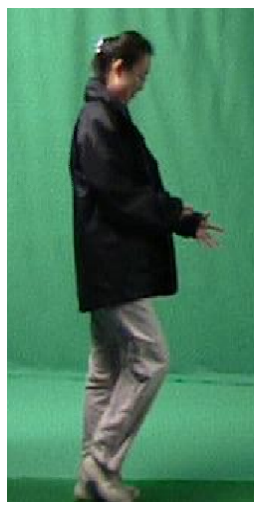

(b)

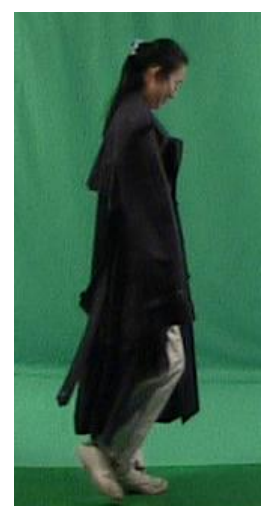

(c)
Figure 3. Clothing Covariate Factors: (a) Normal Clothing (b) Coat (c) Trench Coat

In order to investigate the effects of clothing on the human gait and people identification using gait, we have performed a number of experiments on people wearing different clothing including coat, trench coat and their normal clothing as depicted in Figure (3.2). For each of the clothing-related factors, the gait signature is derived from the dynamic features for 10 subjects with 4 sequences for every individual. The classification performance is assessed as the same way as the footwear case by matching the probe set against the gallery dataset. Figure (4) shows the cumulative match score curves for the gait classification experiments. The correct classification rate for the coat is almost the same as the normal case with reported rates of $83.33 \%$ and $86.67 \%$ respectively. However for the case of the trench coat, the recognition decreases largely to $60 \%$. This is mainly due to nature of the clothing which is distracting the gait dynamics as well as the occlusion of the knee and hip joints faced during the extraction of gait features. This does not occur with the trousers which adhere to the front of the leg, but could equally occur with the female clothing (eg. a chardor ).

\subsection{Load Carriage}

The impact of load carried on human gait and body posture has been extensively investigated for different purposes including medical, training and military [8] use but rarely for the purpose of gait recognition. In [12], Pascoe et al carried out a number of experiments to examine the effects of carrying bags on gait kinematics for youth people. Pascoe reported that the stride length decreases whilst the gait

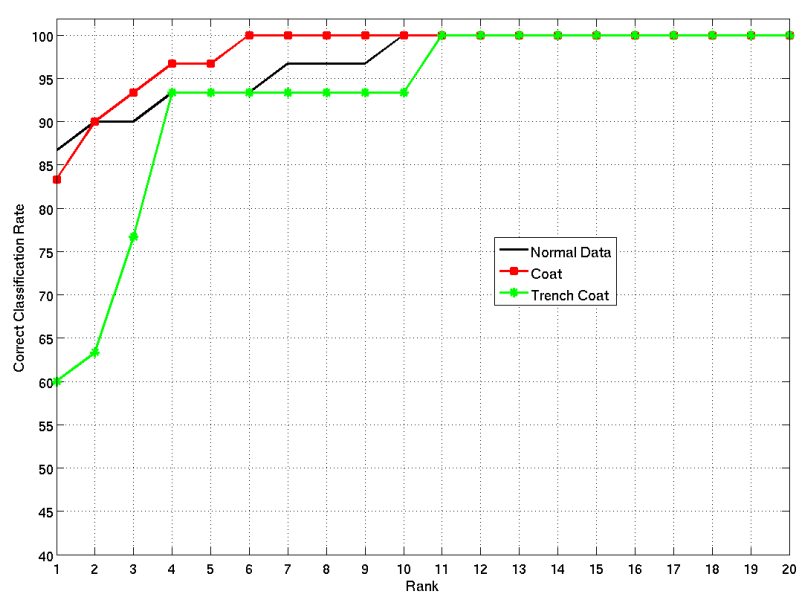

Figure 4. Classification Results for the Clothing Covariates.

cadence increases in response to the weight of the load. The same results were also confirmed by the work of Attwells $e t$ al [2] and Wang [22]. Attwells observed from experiments carried out on military personnel that the gait angular data including the knee and femur angles are significantly affected with the increase of carriage load. For the effects of carrying conditions on the performance of gait recognition, Phillips et al. [15] reported a correct classification rate of $61 \%$ using $K N N$ for $k=1$ employing a silhouette-based method for people carrying a briefcase.

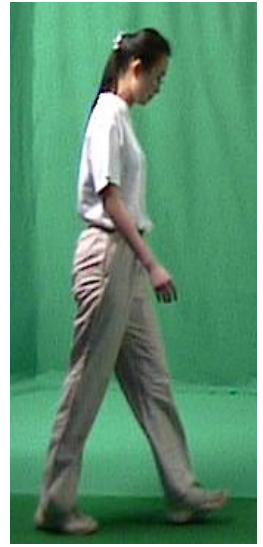

(a)

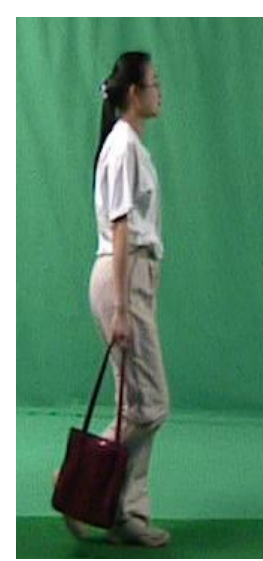

(b)

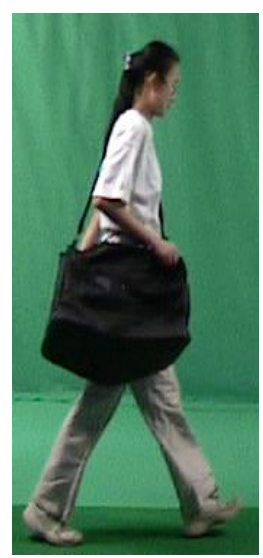

(c)
Figure 5. Load Carriage Covariate Factors: (a) Normal Walking (b) Handbag (c) Barrel Bag

To investigate the impact of load carriage on the performance of gait recognition using the model-based method for the extraction of dynamic gait features, three different covariate cases related to carrying conditions are used to construct the probe dataset. The cases include people carrying handbags and barrel bags besides the normal walking without carriage as illustrated in Figure (5). The probe set consists of 120 video sequences for 10 different sub- 
jects with 4 trials for every case. People in the probe set are matched against the same gallery dataset which is used for the evaluation of previous covariate factors. The classification results for gait recognition are detailed using the CMS curves shown in Figure (7).

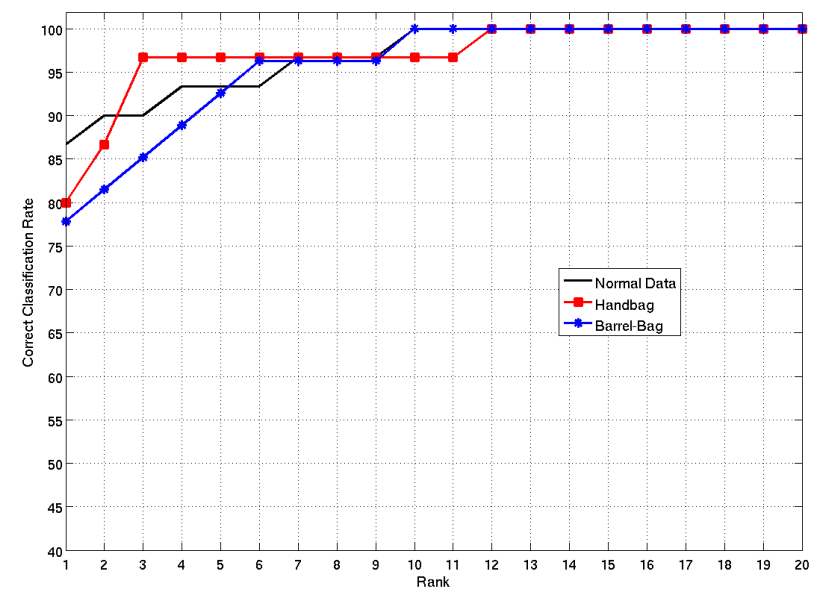

Figure 6. Classification Results for the Load Carriage.

The achieved recognition rate for people carrying a handbag is almost the same as the normal case with a reported CCR of $80 \%$. This is because of the lightness of the handbag which does not affect the gait pattern. For the case of the barrel bag which is covering the mid part of the human body, the recognition rate drops slightly to $77 \%$. However, such results may not express the real impact of the carriage load on the performance of gait recognition. This is because the duration of load carriage was brief, as the responses and effects of load may change with the duration of carriage as a result of exacerbated fatigue. This was not possible to study in this research due to the limitation of the gait database.

\subsection{The Speed Effects}

There is currently not much work that investigates the effects of speed on the performance of gait recognition methods and the relationship between the gait features and the varying walking speed [18]. Based on a model-based method for feature extraction, Yam [23] reported the possible existence of an individual mapping between the walking and running gait patterns. In [17], Bobick et al observed that appearance-based features derived from silhouette of walking people are speed-dependent and therefore, a preprocessing stage for feature adjustment is suggested to improve the recognition performance. To study the impact of speed variation on gait recognition, a probe dataset is constructed consisting of 10 subjects recorded at different walking speed: slow, normal and quick with 4 trials for every case. The recognition rate for both slow and quick walking drops largely to $60 \%$ and $50 \%$ respectively compared

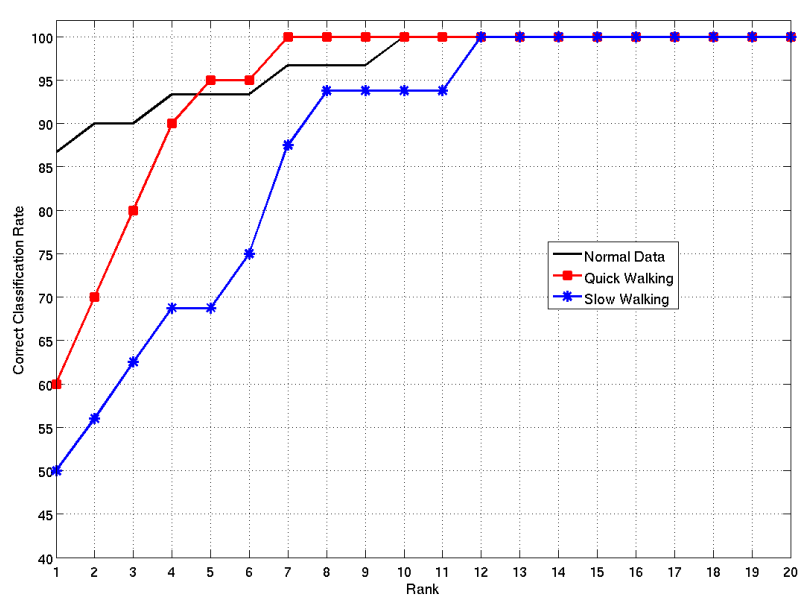

Figure 7. Classification Results for the Walking Speed Covariates.

to the achieved CCR of $\% 86$ for the normal walking case leading to the conclusion that dynamic gait features are also dependent on speed.

\section{Covariate Factor Analysis of Gait Features}

Feature analysis is performed to quantify the footwear effects on the different dynamic gait components employed for recognition. For each of the gait angular signature components ( i.e. knee, ankle and hip), the correct recognition rate is computed using leave-one-out validation and a $K N N$ classifier with $k=5$ for the different covariate factors. The overall results are summarised in Table (1) which shows the means and standard deviations of the recognition rates for the various gait dynamic features. The knee is observed to have the highest average CCR whilst it is the most component susceptible to the different covariates with a standard deviation of $14.1 \%$. The ankle has the lowest standard deviation among the angular features. the vertical tipping motion of the trunk ( $\mathrm{Y}$ displacement ) is observed as the most stable features with high average CCR and almost low standard deviation.

Table 1. Statistical Analysis of Gait Features.

\begin{tabular}{|l|c|c|}
\hline & Mean CCR & Std. Deviation \\
\hline Hip & 25 & 12.1 \\
\hline Knee & 27.9 & 14.1 \\
\hline Ankle & 24.1 & 9.6 \\
\hline X Displacement & 15.9 & 7.2 \\
\hline Y Displacement & 23.3 & 7.3 \\
\hline
\end{tabular}

\section{Conclusions}

In this chapter, we have investigated the impact of the different covariate factors on the performance of gait recognition using kinematic-related features. Four different co- 
variates are analysed including footwear, load carriage, clothing and walking speed. Based on a covariate-based probe dataset of 440 samples, a high recognition rate of $73.4 \%$ is achieved using the $K N N$ classifier with $k=5$. This is to conclude that people identification using dynamic gait features is still perceivable with better recognition rate even under the different covariate factors. The footwear, clothing and load carriage covariates are observed to have almost no effects on the performance of gait recognition with similar results when walking with barefeet or without carrying bags. However, the gait recognition drops largely when walking with flip flops or wearing a trench coat due the difficulties encountered during the extraction of dynamic gait features using the model-based method.

\section{References}

[1] A. S. V. Aguado, M. S. Nixon, and M. E. Montiel. Parameterizing Arbitrary Shapes via Fourier Descriptors for EvidenceGathering Extraction. Computer Vision and Image Understanding, 69(2):202-221, 1998. 2

[2] R. Attwells, S. Birrell, R. Hooper, and N. Mansfield. Influence of Carrying Heavy Loads on Soldiers' Posture, Movements and Gait. Ergonomics, 49(14):1527-1537, 2006. 4

[3] C. BenAbdelkader, R. Cutler, and L. Davis. Person Identification using Automatic Height and Stride Estimation. In Procedings of the International Conference on Pattern Recognition, 4:377-380, 2002. 1

[4] I. Bouchrika and M. S. Nixon. Model-Based Feature Extraction for Gait Analysis and Recognition. In Proceedings of Mirage: Computer Vision / Computer Graphics Collaboration Techniques and Applications, pages 150-160, 2007. 2

[5] R. J. Dobbs, A. Charlett, S. G. Bowes, C. J. A. O'neill, C. Weller, J. Hughes, and S. M. Dobbs. Is This Walk Normal? Age and Ageing, 22(1):27, 1993. 3

[6] W. E. Egan, D. P. Fisher, L. D. Gerber, B. S. Hatler, G. P. Ernst, C. Hall, and N. Henderson. Postural Sway of Subjects Wearing the US Army Chemical Protective Ensemble after Functional Activity. Aviat Space Environ Med, 72(9):831-5, 2001. 3

[7] J. E. Herrero-Jaraba, C. Orrite-Uruñuela, D. Buldain, and A. Roy-Yarza. Human Recognition by Gait Analysis Using Neural Networks. Proceedings of the International Conference on Artificial Neural Networks, 2415:346-369, 2002. 1

[8] J. Knapik, E. Harman, and K. Reynolds. Load Carriage using Packs: A Review of Physiological, Biomechanical and Medical Aspects. Applied Ergonomics, 27(3):207-216, 1996. 4

[9] M. S. Nixon and J. N. Carter. Advances in Automatic Gait Recognition. In Proceedings. Sixth IEEE International Conference on Automatic Face and Gesture Recognition, pages 139-144, 2004. 1

[10] M. S. Nixon, T. N. Tan, and R. Chellappa. Human Identification Based on Gait (The Kluwer International Series on Biometrics). Springer-Verlag New York, Inc. Secaucus, NJ, USA, 2005. 1
[11] M. A. Nurse, M. Hulliger, J. M. Wakeling, B. M. Nigg, and D. J. Stefanyshyn. Changing the Texture of Footwear can Alter Gait Patterns. Journal of Electromyography \& Kinesiology, 15(5):496-506, 2005. 3

[12] D. D. Pascoe, D. E. Pascoe, Y. T. Wang, D. Shim, and C. K. Kim. Influence of Carrying Book Bags on Gait Cycle and Posture of Youths. Ergonomics, 40(6):631-640, 1997. 4

[13] A. Punakallio, S. Lusa, and R. Luukkonen. Protective Equipment Affects Balance Abilities Differently in Younger and Older Firefighters. Aviation, Space, and Environmental Medicine, 74(11):1151-1156, 2003. 3

[14] S. Rahmatalla, H. Kim, M. Shanahan, and C. C. Swan. Effect of Restrictive Clothing on Balance and Gait using Motion Capture and Dynamic Analysis. SAE Transactions Journal of Passenger Cars: Electronic and Electrical Systems, 114:713-722, 2005. 3

[15] S. Sarkar, P. J. Phillips, Z. Liu, I. R. Vega, P. Grother, and K. W. Bowyer. The humanID Gait Challenge Problem: Data Sets, Performance, and Analysis. IEEE Transactions on Pattern Analysis and Machine Intelligence, 27(2):162-177, 2005. 1, 2, 3, 4

[16] P. Somol, P. Pudil, J. Novovičová, and P. Paclík. Adaptive Floating Search Methods in Feature Selection. Pattern Recognition Letters, 20(11-13):1157-1163, 1999. 2

[17] R. Tanawongsuwan and A. Bobick. Modelling the Effects of Walking Speed on Appearance-Based Gait Recognition. in Proceedings of the 2004 IEEE Computer Society Conference on Computer Vision and Pattern Recognition, 2. 5

[18] R. Tanawongsuwan and A. Bobick. Performance Analysis of Time-Distance Gait Parameters under Different Speeds. International Conference on Audio-and Video-Based Biometric Person Authentication, pages 715-724, 2003. 5

[19] G. V. Veres, M. S. Nixon, and J. N. Carter. Modelling the Time-Variant Covariates for Gait Recognition. in Proceedings of 5th International conference on Audio-and VideoBased Viometric Person Authentication, pages 597-606, 2005. 3

[20] G. V. Veres, M. S. Nixon, L. Middleton, and J. N. Carter. Fusion of Dynamic and Static Features for Gait Recognition over Time. In Proceedings of 7th International Conference on Information Fusion, 2, 2005. 3

[21] L. Wang, W. Hu, and T. Tan. A New Attempt to Gait-Based Human Identification. In Proceedings of the International Conference on Pattern Recognition, 1:115-118, 2002. 1

[22] Y. T. Wang, D. D. Pascoe, and W. Weimar. Evaluation of Book Backpack Load during Walking. Ergonomics, 44(9):858-869, 2001. 4

[23] C. Y. Yam, M. S. Nixon, and J. N. Carter. On the Relationship of Human Walking and Running: Automatic Person Identification by Gait. in Proceedings of the International Conference on Pattern Recognition, 2002. 5 